\title{
SARS-CoV envelope protein palmitoylation or nucleocapid association is not required for promoting virus-like particle production
}

\author{
Ying-Tzu Tseng ${ }^{1}$, Shiu-Mei Wang ${ }^{1,2}$, Kuo-Jung Huang ${ }^{1}$ and Chin-Tien Wang ${ }^{1,2^{*}}$
}

\begin{abstract}
Background: Coronavirus membrane $(\mathrm{M})$ proteins are capable of interacting with nucleocapsid $(\mathrm{N})$ and envelope (E) proteins. Severe acute respiratory syndrome coronavirus (SARS-CoV) M co-expression with either N or E is sufficient for producing virus-like particles (VLPs), although at a lower level compared to M, N and E co-expression. Whether $\mathrm{E}$ can release from cells or E/N interaction exists so as to contribute to enhanced VLP production is unknown. It also remains to be determined whether E palmitoylation or disulfide bond formation plays a role in SARS-CoV virus assembly.

Results: SARS-CoV N is released from cells through an association with E protein-containing vesicles. Further analysis suggests that domains involved in E/N interaction are largely located in both carboxyl-terminal regions. Changing all three $\mathrm{E}$ cysteine residues to alanines did not exert negative effects on $\mathrm{E}$ release, $\mathrm{E}$ association with $\mathrm{N}$, or E enhancement of VLP production, suggesting that E palmitoylation modification or disulfide bond formation is not required for SARS-CoV virus assembly. We found that removal of the last E carboxyl-terminal residue markedly affected $\mathrm{E}$ release, $\mathrm{N}$ association, and VLP incorporation, but did not significantly compromise the contribution of $\mathrm{E}$ to efficient VLP production.
\end{abstract}

Conclusions: The independence of the SARS-CoV E enhancement effect on VLP production from its viral packaging capacity suggests a distinct SARS-CoV E role in virus assembly.

\section{Background}

Coronaviruses are enveloped viruses with $27-32 \mathrm{~kb}$ single-strand positive-sense RNA genomes encoding four structural proteins: nucleocapsid $(\mathrm{N})$, spike $(\mathrm{S})$, membrane $(\mathrm{M})$ and envelope $(\mathrm{E})[1,2]$. Translated on free polysomes, highly basic $\mathrm{N}$ interacts with newly synthesized viral genomic RNA to form helical nucleocapsids $[3,4]$. The $\mathrm{M}, \mathrm{S}$ and $\mathrm{E}$ viral membrane proteins are translated on membrane-bound polysomes, inserted into the endoplasmic reticulum (ER), and transported to the ER-Golgi intermediate compartment (ERGIC), where E and $\mathrm{M}$ interact and trigger budding $[5,6] . \mathrm{N}$ and $\mathrm{S}$ are incorporated into virions via interaction with $\mathrm{M}$, with virions accumulating in large, smooth-walled vesicles that

\footnotetext{
* Correspondence: chintien@ym.edu.tw

'Department of Medical Research, Taipei Veterans General Hospital, 201, Sec. 2, Shih-Pai Road, Taipei 11217, Taiwan

${ }^{2}$ Institute of Clinical Medicine, National Yang-Ming University School of Medicine, 201, Sec. 2, Shih-Pai Road, Taipei 11217, Taiwan
}

eventually fuse with the plasma membrane and release virions from cells [2,7-11].

Coronavirus $\mathrm{E}$ is a small integral membrane protein consisting of approximately 76 to 109 amino acids and containing a hydrophobic domain. Several researchers have suggested that coronavirus $E$ functions as an ion channel $[12,13]$. The role of the coronavirus $\mathrm{E}$ ion channel in the virus life cycle is not completely clear. The addition of hexamethylene amiloride (HMA, an ion channel inhibitor of mouse hepatitis coronavirus [MHV] and human coronavirus 229E [HCoV229E] ion channel activity in vitro) to culture medium blocks MHV and HCoV229E replication [12], suggesting that the coronavirus $\mathrm{E}$ ion channel plays a role in virus replication. Two or three cysteine residues are located on the carboxyl side of the hydrophobic domain in coronavirus $\mathrm{E}$ proteins, with some serving as targets for palmitoylation [14-17], which may contribute to virus assembly in the MHV. 
E plus $M$ has been shown to be sufficient for VLP formation in the MHV [18], transmissible gastroenteritis virus (TGEV) [19], bovine coronavirus (BCoV) [19], infectious bronchitis virus (IBV) [5], and SARS-CoV [20]. Although $\mathrm{M}$ and $\mathrm{N}$ co-expression is also sufficient for SARS-CoV VLP production [21,22], VLP yields are further enhanced by E co-expression [23]. According to these data, SARS-CoV E plays a supporting role in promoting virus assembly and/or budding. $\mathrm{E}$ and $\mathrm{N}$ are thought to be packaged into VLPs through separate and individual associations with $\mathrm{M}$; it remains unknown whether $\mathrm{E} / \mathrm{N}$ interaction exists, which might contribute to enhanced virion production. MHV and IBV E proteins are capable of release from cells as vesicles [5,24], implying a relationship between $\mathrm{E}$ release and its contribution to virus production. Our goal for this study was to determine whether SARS-CoV $\mathrm{N}$ is capable of interacting with $\mathrm{E}$ and cell release via an association with $\mathrm{E}$ containing vesicles. Our results indicate no correlation between SARS-CoV E capacity to release or interact with $\mathrm{N}$, or its ability to promote VLP production.

\section{Methods}

Plasmid construction

Mammalian expression vectors encoding SARS-CoV $M$, N, S and E were provided by G. J. Nabel [21]. A pair of upstream and downstream primers was used to amplify Ecoding fragments via PCR-based overlap extension mutagenesis [25]. Primers used to introduce an HA or FLAG epitope tag to the $\mathrm{E}$ amino or carboxyl terminus are 5' -TTCTGCGATATCGCCACCATGTACCCATACGAC GTGCCTGACTACGCCTACAGCTTCGTGAGCG-3' (containing a flanking EcoRV restriction site and HA tagcoding nucleotides) and 5'-GCGGATCCTCACTTGTC GTCGTCGTCCTTGTAGTCGCCCACCAGCAGGTCG GGCAC-3' (containing a flanking BamHI restriction site and FLAG tag-coding nucleotides). The forward primer is $5^{\prime}$-GTCTGAGCAGTACTCGTTGCTG-3 (referred to as the $\mathrm{N}$ primer) and reverse primer $5^{\prime}$-GGAAAGGA CAGTGGGAGTGGCAC-3' (referred to as the anti-N primer). Primers used to construct designated E mutants were EC3A, 5' - CTGAGGCTGGCCGCATATGCCGCG AACATCGTGAACGTGAGC-3' (reverse); 74LL/AA,5' GCAGATCTGGATCCTAGTTCACACGGCCGCGTCG GGCACGCC-3' (reverse); E $\triangle 76 \mathrm{~V}, 5^{\prime}$ - CAGATCTGGA TCCTAGTTCACAGCAGGTCGGGCAC-3' (reverse). The $\mathrm{N}$ primer serves as a forward primer while the anti$\mathrm{N}$ primer was used as a reverse primer for the second round PCR amplification. Purified PCR product was digested with BamHI and EcoRV and ligated into the SARS-CoV E expression vector. When constructing NDsRed, the $\mathrm{N}$ primer served as the forward primer, using the SARS-CoV N expression vector as a template and 5' - GCGGATCCTGGGTGCTGTCGGCGCTG-3' as the reverse primer. Amplified PCR product was digested with BamHI and SalI and ligated into pDsRedMonomer-N1 (Clontech). The cloned N-DsRed was digested with NheI and BamHI and ligated into pEGFPN1 (Clonetech), yielding construct N-EGFP.

GST-M was constructed by digesting $M$ expression vector with EcoRV and NotI, ligated into pCDNA3.1myc-HisA (Invitrogen). The resultant construct was then digested with BamHI and Not1, and then the $M$ coding sequence fragment was fused to carboxyl terminus of GST, which is directed by a mammalian elongation factor Ia promoter [26]. GST-N fusions, as described previously [27], were constructed by fusing SARS-CoV N coding sequences to the carboxyl terminus of GST.

\section{Cell culture and transfection}

$293 \mathrm{~T}$ and HeLa cells were maintained in Dulbecco's modified Eagle's medium (DMEM) supplemented with $10 \%$ fetal calf serum (GIBCO). Confluent cells were trypsinized and split 1:10 onto $10 \mathrm{~cm}$ dishes $24 \mathrm{~h}$ prior to transfection. For each construct, cells were transfected with $20 \mu \mathrm{g}$ of plasmid DNA using the calcium phosphate precipitation method; $50 \mu \mathrm{m}$ chloroquine was added to enhance transfection efficiency. Unless otherwise indicated, $10 \mu \mathrm{g}$ of each plasmid was used for co-transfection. Culture supernatant and cells were harvested for protein analysis 2-3 d post-transfection. For HeLa transfection, plasmid DNA was mixed with GenCarrier (Epoch Biolabs) at a ratio of $1 \mu \mathrm{g}$ to $1 \mu \mathrm{l}$; the transfection procedure was performed according to the manufacturer's protocols.

\section{Western immunoblot}

At 48-72 h post-transfection, supernatant from transfected cells was collected, filtered, and centrifuged through $2 \mathrm{ml}$ of $20 \%$ sucrose in TSE $(10 \mathrm{mM}$ Tris- $\mathrm{HCl}$ [pH 7.5], $100 \mathrm{mM} \mathrm{NaCl}, 1 \mathrm{mM}$ EDTA plus $0.1 \mathrm{mM}$ phenylmethylsulfonyl fluoride [PMSF]) at $4^{\circ} \mathrm{C}$ for $40 \mathrm{~min}$ at $274,000 \times g$. Pellets were suspended in IPB (20 mM Tris- $\mathrm{HCl}$ [pH 7.5], $150 \mathrm{mM} \mathrm{NaCl}, 1 \mathrm{mM}$ EDTA, $0.1 \%$ SDS, $0.5 \%$ sodium deoxycholate, $1 \%$ Triton X-100, $0.02 \%$ sodium azide) plus $0.1 \mathrm{mM}$ PMSF. Cells were rinsed with ice-cold phosphate-buffered saline (PBS), collected in IPB plus $0.1 \mathrm{mM}$ PMSF, and microcentrifuged at $4{ }^{\circ} \mathrm{C}$ for $15 \mathrm{~min}$ at $13,700 \times \mathrm{g}$ to remove unbroken cells and debris. Supernatant and cell samples were mixed with equal volumes of $2 \mathrm{X}$ sample buffer $(12.5 \mathrm{mM}$ Tris- $\mathrm{HCl}$ [pH 6.8], 2\% SDS, 20\% glycerol, $0.25 \%$ bromphenol blue) and $5 \% \beta$-mercaptoethanol and boiled for $5 \mathrm{~min}$ or (for the M-containing samples) incubated at $45^{\circ} \mathrm{C}$ for $10 \mathrm{~min}$. Samples were resolved by electrophoresis on SDS-polyacrylamide gels and electroblotted onto nitrocellulose membranes. Membrane-bound M, M-FLAG, HA-E, E-FLAG or GST proteins were immunodetected using a SARS-CoV $\mathrm{M}$ rabbit anitserum, anti-HA (LTK 
BioLaboratories, Taiwan), anti-FLAG or anti-GST(Sigma) monoclonal antibody at a dilution of 1:1,000. For SARS-CoV N or S detection, a mouse monoclonal antibody $[28,29]$ was used at a dilution of 1:5,000. The secondary antibody was a sheep anti-mouse or donkey anti-rabbit horseradish peroxidase-(HRP) conjugated antibody (Invitrogen), both at 1:5,000 dilutions.

\section{Laser scanning immunofluorescence microscopy}

HeLa cells were split 1:80 onto coverslips $24 \mathrm{~h}$ before transfection. Between 18 and 24 h post-transfection, , cells were washed with PBS and permeabilized at room temperature for $10 \mathrm{~min}$ in $\mathrm{PBS}$ plus $0.1 \%$ Triton X-100 following fixation at $4^{\circ} \mathrm{C}$ for $20 \mathrm{~min}$ with methanol/acetone (1:1). Samples were incubated with the primary antibody for $1 \mathrm{~h}$ and with the secondary antibody for $30 \mathrm{~min}$. Following each incubation, samples were subjected to three washes (5 to $10 \mathrm{~min}$ each) with DMEM/calf serum. Primary antibody concentrations were anti-HA at a dilution of 1:500. A rabbit anti-mouse rhodamine-conjugated antibody at a 1:100 dilution served as the secondary antibody (Cappel, ICN Pharmaceuticals, Aurora, OH). After a final DMEM/calf serum wash, the coverslips were washed three times with PBS and mounted in 50\% glycerol in PBS for viewing. Images were analyzed and photographs taken using the inverted laser Zeiss.

\section{lodixanol density gradient fractionation}

Supernatants from transfected 293 T cells were collected, filtered, and centrifuged through $2 \mathrm{ml} 20 \%$ sucrose cushions as described above. Viral pellets were suspended in PBS buffer and laid on top of a pre-made $10-40 \%$ iodixanol (OptiPrep) gradient consisting of $1.25 \mathrm{ml}$ layers of $10,20,30$ and $40 \%$ iodixanol solution prepared according to the manufacturer's instructions (Axis-Shield, Norway). Gradients were centrifuged in a SW50.1 rotor at $40,000 \mathrm{rpm}$ for $16 \mathrm{~h}$ at $4^{\circ} \mathrm{C} ; 500 \mu \mathrm{l}$ fractions were collected from top to bottom and densities were measured for each. Proteins in each fraction were precipitated with $10 \%$ trichloroacetic acid (TCA) and subjected to Western immunoblotting.

\section{GST pull-down assay}

GST pull-down protocols were as previously described [27]. Briefly, $500 \mu \mathrm{l}$ of PNS containing complete protease inhibitor cocktail was mixed with $30 \mu \mathrm{l}$ of glutathione agarose beads (Sigma). All reactions took place at $4^{\circ} \mathrm{C}$ overnight on a rocking mixer. Immunoprecipitateassociated resin or bead-bound complexes were pelleted, washed tree times with lysis buffer, two times with PBS, eluted with $1 \mathrm{X}$ sample buffer, and subjected to SDS-10\% PAGE as described above.

\section{Results}

SARS-CoV $\mathrm{E}$ is capable of associating with $\mathrm{N}$

In a previous study we found that SARS-CoV $\mathrm{M}$ coexpression with $\mathrm{N}$ leads to VLP formation, and that a FLAG tagged at the SARS-CoV $M$ carboxyl terminus (M-FLAG) significantly inhibits $\mathrm{N}$ packaging into VLPs [22]. Since N release into medium depends on an association with $\mathrm{M}$, and since $\mathrm{E}$ may promote VLP production, we set out to confirm whether E complements M-FLAG in $\mathrm{N}$ packaging into VLPs. Toward this goal we coexpressed $\mathrm{N}$ with M-FLAG in the absence or presence of SARS-CoV E tagged with either $\mathrm{HA}$ at the amino terminus (HA-E) or with FLAG at the carboxyl terminus (E-FLAG). As shown in Figure 1, $\mathrm{N}$ that was barely detectable in medium became readily detected following co-expression with HA-E (lanes 9 versus 10). Since N is incapable of associating with M-FLAG, a simple explanation would be the ability of $\mathrm{E}$ to promote $\mathrm{N}$ incorporation into VLPs via E/N interaction. E-FLAG was barely

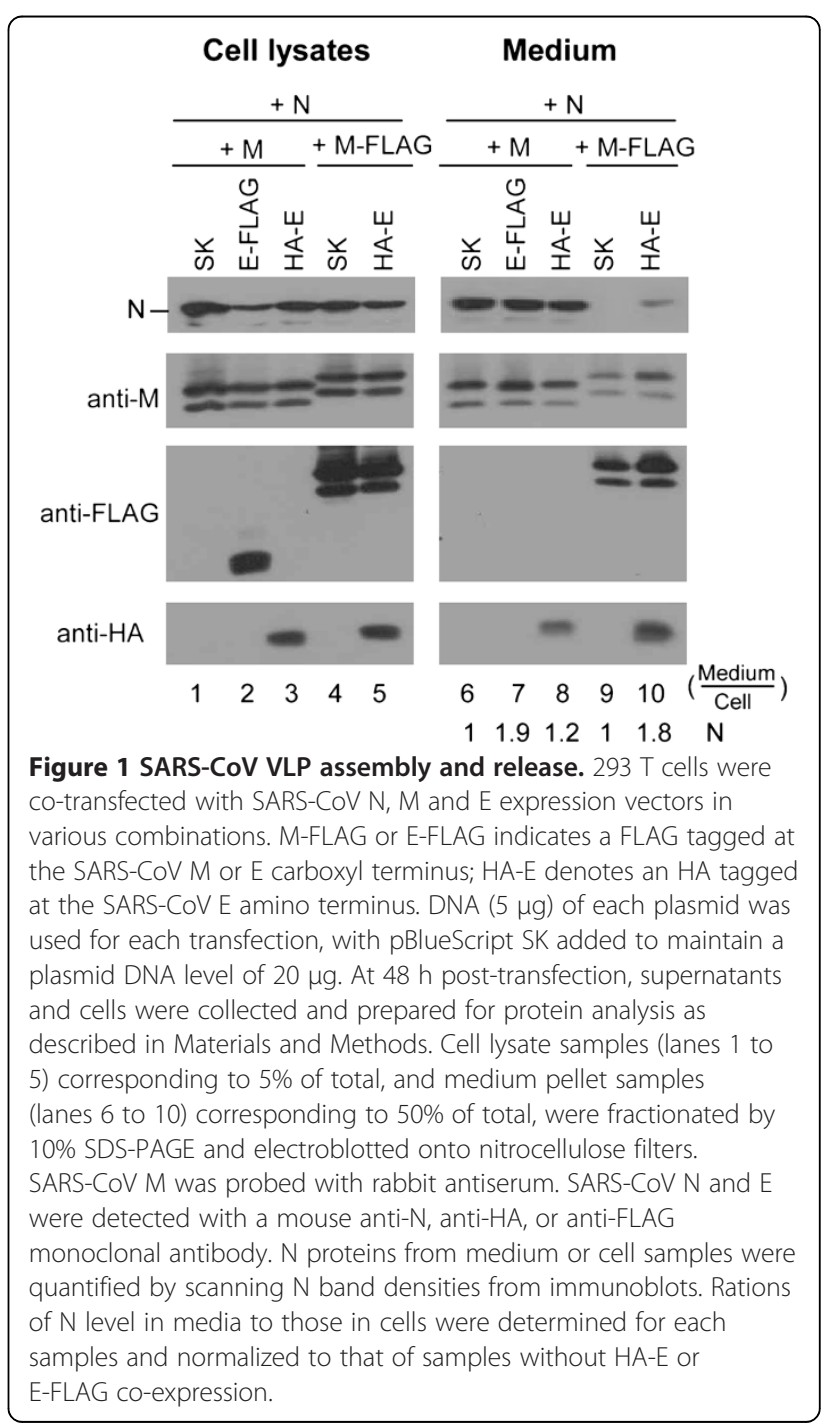


detected in medium (Figure 1, lane 7), suggesting that the FLAG tagged at the E carboxyl terminus affected $\mathrm{E}$ release or $\mathrm{E}$ association with $\mathrm{M}$ and/or $\mathrm{N}$. To confirm that E-mediated $\mathrm{N}$ release is not due to HA tag, we coexpressed $\mathrm{N}$ with $\mathrm{E}$ and found that $\mathrm{E}$ without $\mathrm{HA}$ tag can still promote $\mathrm{N}$ release (data not shown), suggesting that HA tag exerts no major impacts on E-mediated $\mathrm{N}$ release.

Since SARS-CoV E and $M$ are both capable of release into medium, $\mathrm{N}$ detected in medium may be a result of an association with released M-FLAG in the presence of $\mathrm{E}$, but not a result of direct $\mathrm{E} / \mathrm{N}$ interaction. To rule out this possibility and to confirm that a specific N-E interaction exists, we co-expressed $\mathrm{N}$ and either HA-E, EFLAG, $\mathrm{M}$ or $\mathrm{S}$. As expected, $\mathrm{N}$ was readily detected when co-expressed with HA-E or with M, but barely detectable in culture medium when expressed alone or coexpressed with S (Figure 2A, lanes 5 and 8) or E-FLAG (which was also barely detectable in medium) (Figure 2B, lane 3). These data support our hypothesis that SARS$\mathrm{CoV} \mathrm{N}$ is capable of release into medium via association with E, and suggests that FLAG tagged at the SARS$\mathrm{CoV}$ E carboxyl terminus significantly reduces $\mathrm{E}$ release capacity in addition to impairing $\mathrm{E}$ incorporation into VLPs.

To confirm that $\mathrm{N}$ release is associated with $\mathrm{E}$ in pelletable particle form, we subjected culture medium from cells expressing E, E plus N, or E plus $\mathrm{N}$ and $\mathrm{M}$ to iodixanol density gradient fractionation, and found that $\mathrm{E}$ primarily sedimented with co-expressed $\mathrm{N}$ at a slightly lower density fraction compared to $\mathrm{E}$ expression alone (Figure 2C). Particles formed by $\mathrm{E}$ or $\mathrm{E}$ plus $\mathrm{N}$ exhibited an iodixanol density of 1.13 to $1.16 \mathrm{~g} / \mathrm{ml}$, whereas VLPs formed by $\mathrm{E}, \mathrm{N}$ and $\mathrm{M}$ displayed densities ranging from 1.11 to $1.13 \mathrm{~g} / \mathrm{ml}$. Combined, these data support the idea that $\mathrm{N}$ released into medium is associated with $\mathrm{E}$ vesicles when co-expressed with $\mathrm{E}$.

\section{RNA is not required for efficient $\mathrm{E} / \mathrm{N}$ interaction}

SARS-CoV N contains an RNA binding domain [30], and results from our previous study indicate that RNA can enhance the N-N interaction [22]. Here our goal was to determine whether the presence of RNA is required for $\mathrm{E} / \mathrm{N}$ interaction. HA-E or $\mathrm{N}$ was co-expressed with GST tagged at the $\mathrm{N}$ amino terminus (GST-N). E or N association with GST-N was assessed using a GST pull-down assay in the presence or absence of RNase. GST by itself was not capable of pulling down HA-E or $\mathrm{N}$ (Figure 3A, lanes 5-6). RNase treatment significantly reduced levels of co-pulled-down N (Figure 3A, lane 9), which is consistent with past results [22]. However, the same RNase condition did not reduce the amount of copulled-down HA-E, suggesting that RNA is not required for $\mathrm{E} / \mathrm{N}$ interaction. We frequently observed slight increases in pulled-down HA-E following treatment with RNase (Figure 3A, lane 10 vs. lane 8). Since RNA binds to $\mathrm{N}$ readily, it is likely that RNA-bound $\mathrm{N}$ molecules may associate less efficiently with $\mathrm{E}$.

\section{E association domain is largely located in the $\mathrm{N}$ carboxyl-terminal region}

To map the $\mathrm{N}$ sequences involved in $\mathrm{E}$ association, we constructed a set of GST-N fusions containing multiple $\mathrm{N}$ coding sequences fused to the GST carboxyl terminus (Figure 3B). Each GST fusion construct was transiently co-expressed with E, and culture supernatants and cell lysates were analyzed by Western immunoblotting. As shown in Figure 3C, all GST fusions except for GST-N7 (containing $\mathrm{N}$ residues 2 to 86 , lane 9) were released into medium when co-expressed with E. None of the GST fusions were readily detected in medium without coexpressed E (data not shown), suggesting that the detected GST-N fusions were released into medium via $\mathrm{E}$ association, and that the $\mathrm{E}$ binding domains are largely located in the $\mathrm{N}$ carboxyl-terminal region (likely involving amino acid residues 87 to 421). Results from a GST pull-down assay (Figure 3D) provide further support for this conclusion.

\section{Cysteine residues are not required for $\mathrm{E}$ release, $\mathrm{E} / \mathrm{N}$ interaction, or $E$ enhancement of VLP production}

SARS-CoV and MHV E proteins are post-translationally modified with palmitic acid. Lack of palmitoylation modification can markedly decrease SARS-CoV E membrane association [16] and MHV E stability [17]. Substitution mutations for cysteines-the palmitoylation targets in MHV E can reduce virus yields, implying their importance for MHV virus production [14,17]. Accordingly, we tried to determine whether equivalent cysteines play a role in SARS-CoV E/N interaction and VLP assembly by constructing an E mutant (designated EC3A) with alanine replacements for SARS-CoV E cysteine residues C40, C43 and C44. Supernatant and cell lysate samples containing HA-E were analyzed under non-reducing conditions to confirm the presence of disulfide bonds between SARS$\mathrm{CoV} \mathrm{E}$ molecules. Results indicate the presence of an $18 \mathrm{kDa}$ band (equivalent to an HA-E dimer) (Figure 4A, lanes 2 and 11, arrowheads), which agrees with a previous report [31]. As expected, only the E monomer was detected in EC3A-containing samples under non-reducing conditions (Figure 4A, lanes 3 and 12).

While most of intracellular E retains its monomeric form, most released $\mathrm{E}$ was detected in dimeric form (Figure 4A, lane 2 vs. lane 11), suggesting that released SARS-CoV E proteins are largely linked by disulfide bonds. Interestingly, EC3A was still efficiently released into medium and apparently capable of promoting $\mathrm{N}$ release (Figure 4A, lanes 14 and 18). The relatively low level of medium HA-E observed 


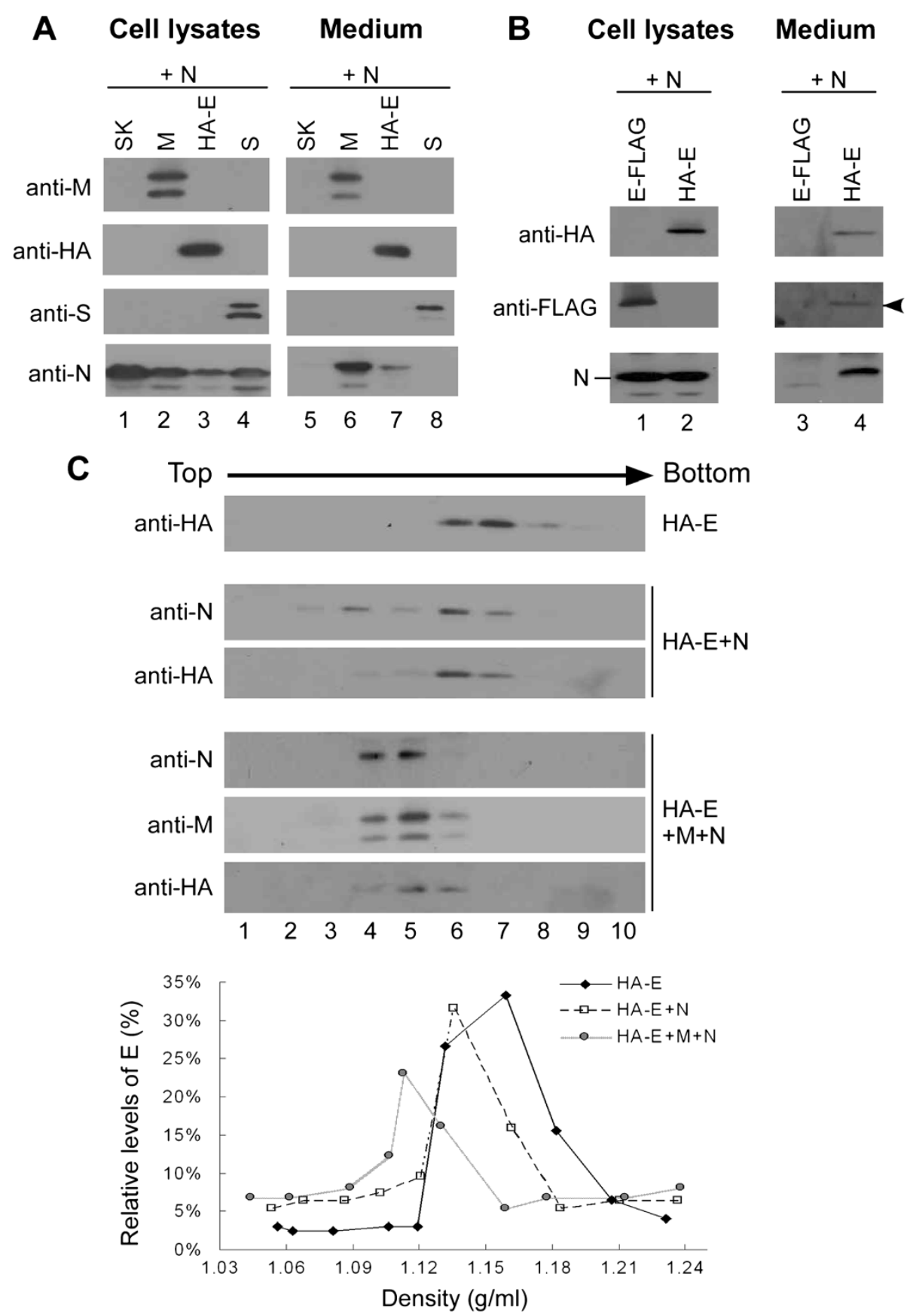

Figure 2 SARS-CoV VLP analysis. (A-B) 293 T cells were co-transfected with E tagged with either a FLAG or HA and N, M or S expression vectors. Culture supernatants were collected $48 \mathrm{~h}$ post-transfection and subjected to Western immunoblotting. E proteins tagged with $\mathrm{HA}$ were probed with an anti-HA antibody. The lane 4 band (panel B, arrowhead) resulted from the incomplete stripping of an anti-HA probe. (C) For buoyant density gradient analysis, concentrated supernatants derived from E, E plus $\mathrm{N}$, or E plus $\mathrm{M}$ and $\mathrm{N}$ transfection samples were centrifuged through a 10-40\% iodixanol gradient for $16 \mathrm{~h}$. Equal quantities of ten fractions were collected from top to bottom. Fraction densities were measured and SARS-CoV, E, M and N proteins analyzed by Western immunoblotting, using anti-HA, anti-M and anti-N antibodies as probes.

in Figure 4A is due to low expression level (top panels, lanes 17 vs. 8). Nevertheless, our data indicate that EC3A produced VLPs as efficiently as wt when co-expressed with both $\mathrm{M}$ and $\mathrm{N}$ (Figure 4B, lanes 10-12), suggesting that SARS-CoV E cysteines are not involved in E release, $\mathrm{E} / \mathrm{N}$ interactions, or VLP assembly. EC3A exhibited greater release efficiency compared to wt, implying that SARS-CoV E cysteines are involved in the E trafficking process. However, immunofluorescence results indicate that the EC3A subcellular distribution pattern was similar to that of the wt. 


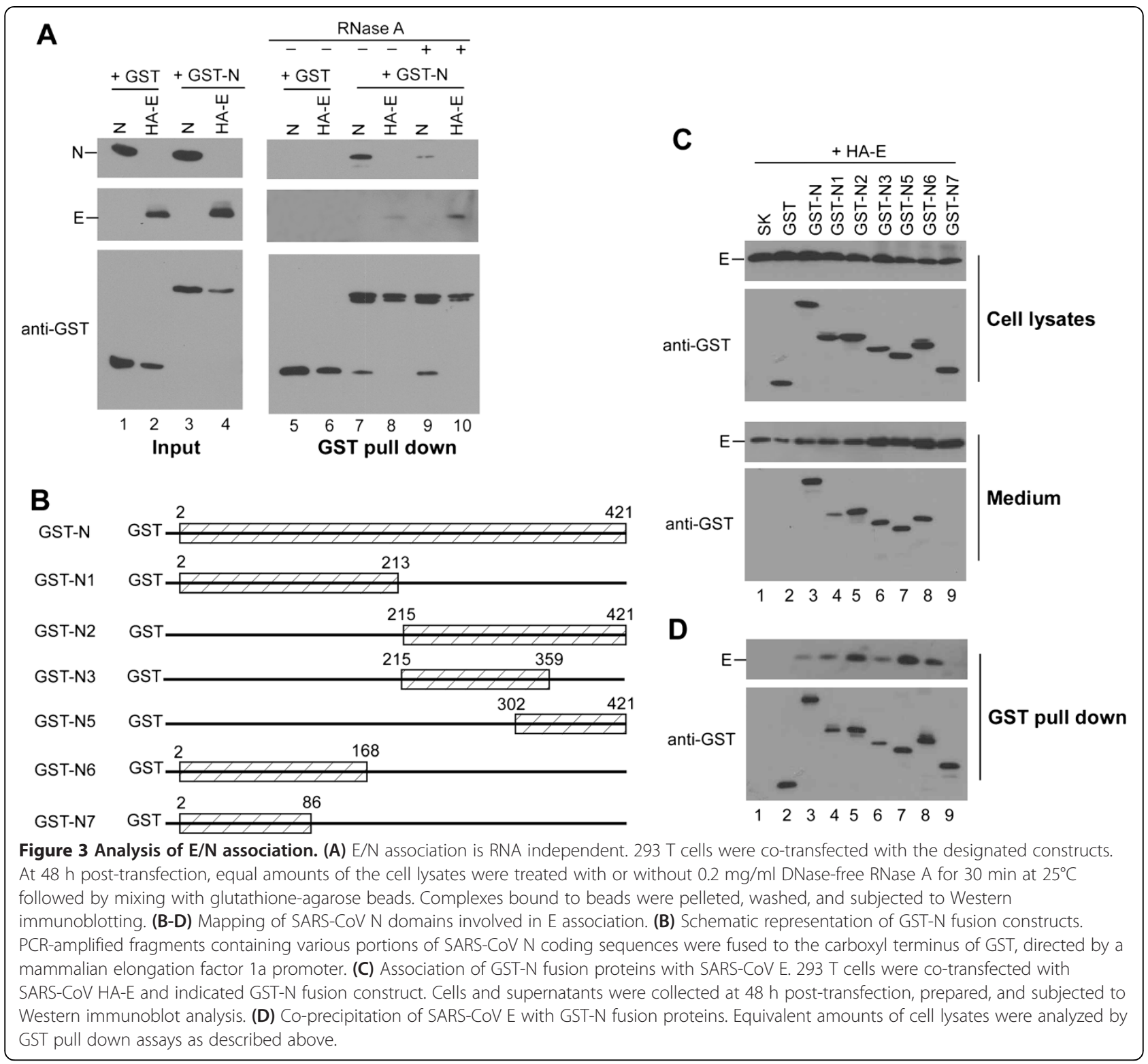

\section{Removal of last amino acid residue from the $E$} carboxyl-terminal tail significantly affects $E$ release and

\section{$\mathrm{E} / \mathrm{N}$ interaction}

E-FLAG was released at a lower efficiency level compared to HA-E, suggesting that the carboxyl-terminal tail domain may be involved in the $\mathrm{E}$ release process. We hypothesized that the E-carboxyl tail is also involved in $\mathrm{E} / \mathrm{N}$ interaction. To test this idea, we constructed two E mutants: E $\Delta 76 \mathrm{~V}$ (with the final carboxyl-terminal residue valine removed) and E74LL/AA (with the carboxylterminal dileucine motif 74-LL-75 replaced with alanines). According to our results, (a) the E74LL/AA mutant was capable of release, and (b) a positive correlation exists between the release levels of co-expressed $\mathrm{N}$ and E74LL/AA (Figure 5A, lanes 10-15). In addition,
E74LL/AA was capable of producing VLPs as efficiently as wt when co-expressed with $\mathrm{M}$ and $\mathrm{N}$ (Figure 5A, lanes 16 vs. 17 and 18). These results suggest that 74LL-75 was not functionally involved in $\mathrm{E}$ release, E/N interaction, or the promotion of VLP production. Conversely, E $\triangle 76 \mathrm{~A}$ was not capable of efficiently promoting $\mathrm{N}$ release, and exhibited a reduced release capacity (Figure 5B, lanes 12 to 15). GST pull-down assay data suggest that the E $\Delta 76 \mathrm{~V}$ mutation markedly affected $\mathrm{E} / \mathrm{N}$ interaction, but exerted no major impacts on N/M association (Figure 5C, lanes 16 vs. 18). The level of GST-Nassociated E was significantly lower than that of GST-Massociated $\mathrm{E}$, suggesting that $\mathrm{E} / \mathrm{N}$ association is not as strong as E/M association (Figure $5 \mathrm{C}$, lanes 15 vs. 17). While pull-down assay results suggest an association 


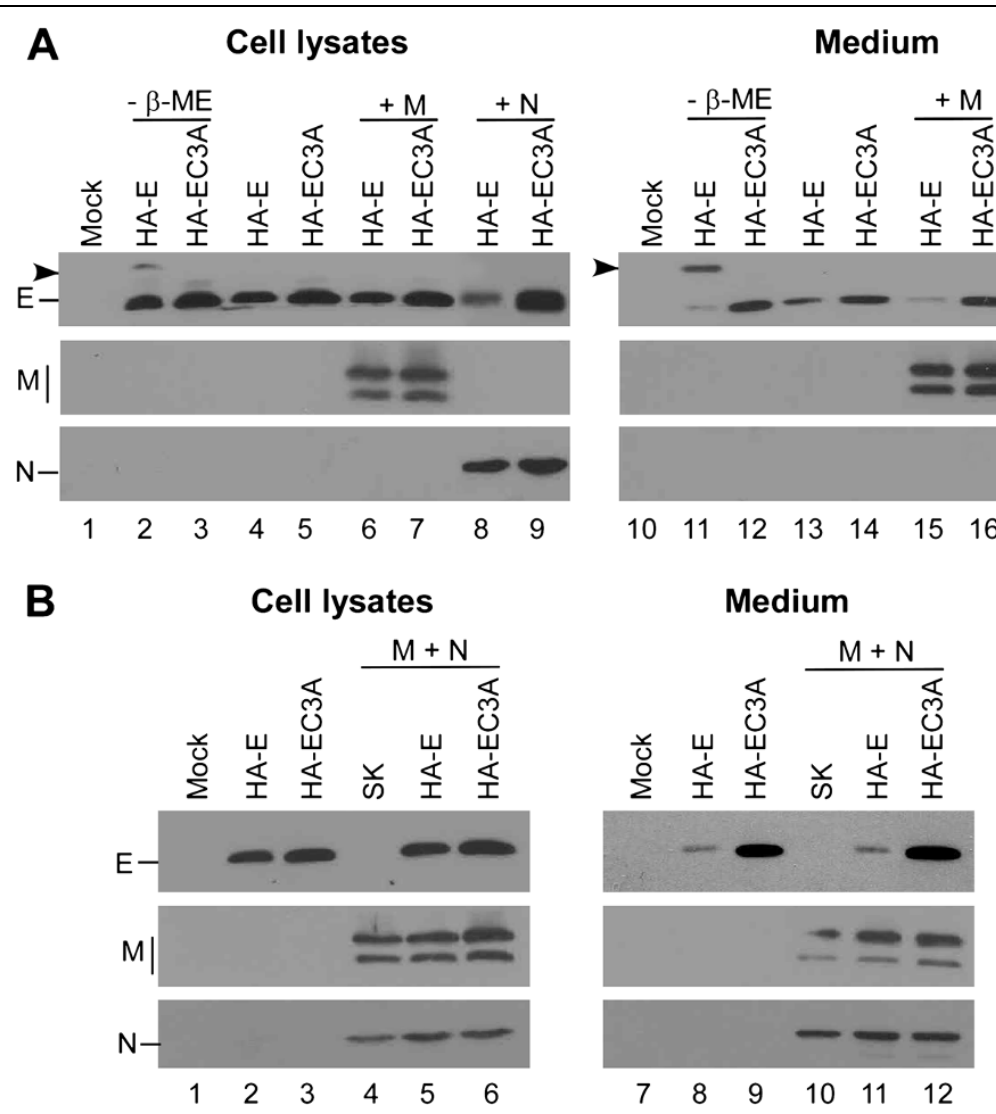

Figure 4 Changing all three cysteine residues to alanines exerted no negative effects on $\mathrm{E}$ release or its capability to interact with $\mathrm{N}$ or VLP production. (A-B) 293 T cells were transfected with SARS-CoV HA-E expression vectors alone, or co-transfected with HA-E plus M and/or N expression vectors. EC3A denotes alanine replacement of all three cysteine residues at E (C40, C43 and C44). Supernatants and cells were collected at $48 \mathrm{~h}$ post-transfection and analyzed by Western immunoblotting. Aliquots of cell and supernatant samples containing E proteins (panel $\mathbf{A}$, lanes 1 to 3 and 10 to 12) were prepared without reducing reagent ( $\beta$-mercaptoethanol). SARS-CoV E, M, and N were respectively probed with anti-HA, anti-M and anti-N antibodies. Arrowheads indicate the position of HA-E dimer.

between $\mathrm{E} \Delta 76 \mathrm{~V}$ and $\mathrm{M}, \mathrm{E} \Delta 76 \mathrm{~V}$ release was not significantly enhanced following $\mathrm{M}$ co-expression (Figure $5 \mathrm{C}$, lane 18 vs. Figure 5B, lanes 17 and 20). A possible explanation is steric hindrance during VLP assembly. Note that $\mathrm{E} \Delta 76 \mathrm{~V}$ was still capable of promoting VLP production as efficiently as the wt, despite defective packaging into VLPs (Figure 5B, lane 20). This suggests that the SARS-CoV E contribution to VLP production is independent of its ability to be packaged into VLPs.

\section{None of the mutations significantly affected SARS-CoV E subcellular localization}

To determine whether mutations affecting E subcellular localization also partly accounted for altered release efficiency, we co-expressed each mutant with a Golgi labeling marker (pECFP-Golgi). According to a confocal microscopy analysis, wt and the EC3A, E74LL/AA, and $\mathrm{E} \triangle 76 \mathrm{~V}$ mutants largely localized in the Golgi area (Figure 6A), suggesting that none of the mutations significantly affected $\mathrm{E}$ subcellular localization. When co-expressed with EGFP-tagged $\mathrm{N}$, a fraction of $\mathrm{N}$ co- localized with $\mathrm{E} \Delta 76 \mathrm{~V}$ in the perinuclear area; this staining pattern was barely distinguishable from that of cells co-expressing HA-E and N-EGFP (Figure 6B). This implies that even though $\mathrm{E} \Delta 76 \mathrm{~V}$ may associate with $\mathrm{N}$ to a certain extent, the association is insufficient for enabling E $\Delta 76 \mathrm{~V}$ co-precipitation with GST-N (Figure 5C).

\section{Discussion}

To our knowledge, this is the first report of interaction between SARS-CoV E and N proteins. Both SARS-CoV spike (S) and $E$ proteins can be released into medium; however, unlike E, $S$ cannot promote the release of coexpressed $\mathrm{N}$ (Figure 2A). This suggests that $\mathrm{N}$ is incapable of associating with $\mathrm{S}$, and supports the idea of specific $\mathrm{E} / \mathrm{N}$ interaction. While RNA can enhance $\mathrm{N} / \mathrm{N}$ interaction, the presence of RNA is not necessary for $\mathrm{E} /$ $\mathrm{N}$ interaction. Genetic analyses suggest that $\mathrm{E}$ binding domains are largely located in the $\mathrm{N}$ carboxyl-terminal region (Figure 3).

SARS-CoV $\mathrm{M}$ or $\mathrm{E}$ by itself can secrete into medium as vesicles but not virus-like particles (VLPs) which can 

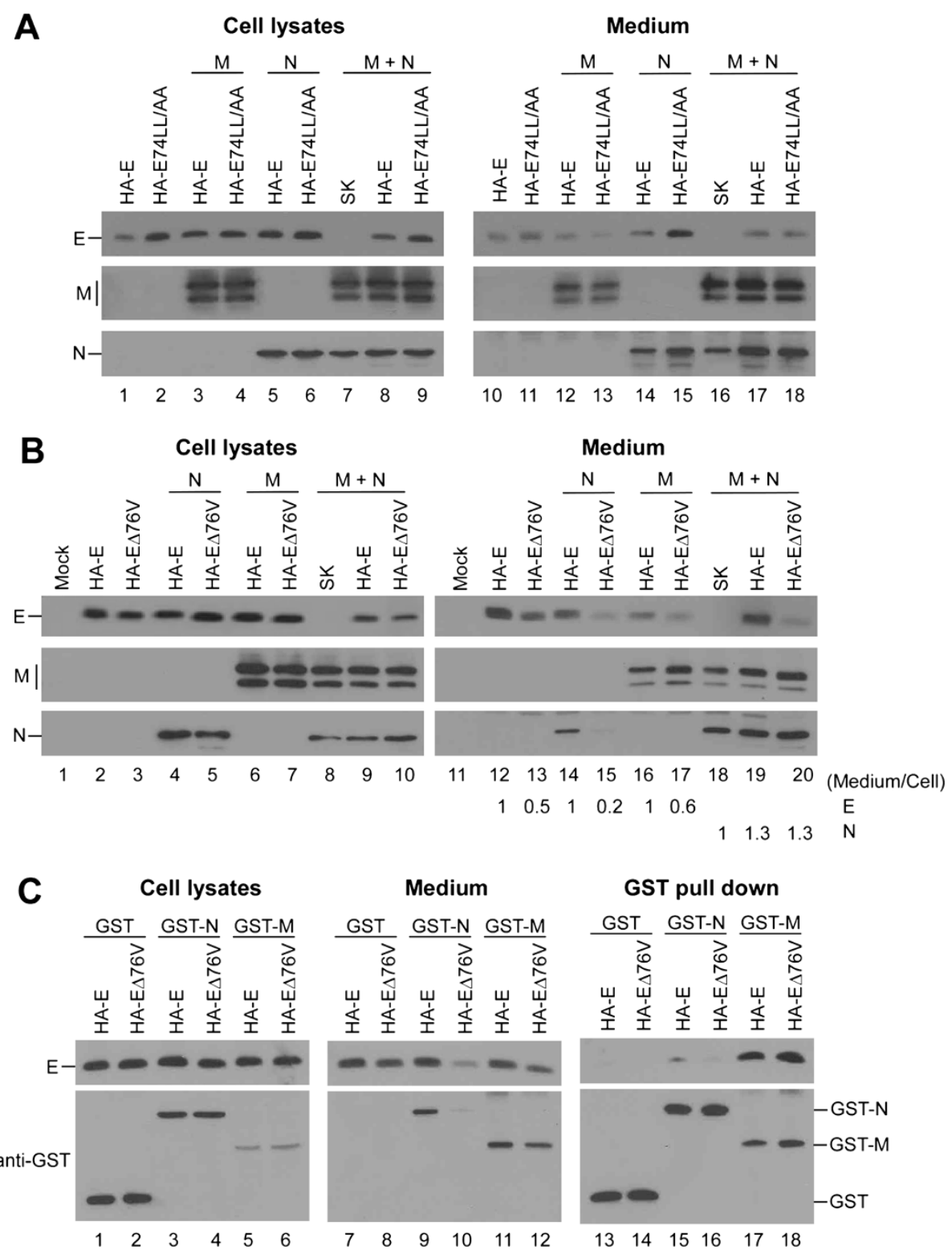

Figure 5 Effects of E carboxyl tail mutations on E/N association and VLP production. (A-B) 293 T cells were transfected with HA-tagged E expression vector alone or in combination with $\mathrm{M}$ and/or $\mathrm{N}$ expression vectors. 74LL/AA indicates alanine substitutions for two leucine residues at the E carboxyl tail (74-LL-75). $\Delta 76 \mathrm{~V}$ denotes a deletion of the last carboxyl-terminal residue V76 from E. Supernatant and cells were harvested at $48 \mathrm{~h}$ post-transfection, prepared, and subjected to Western immunoblotting. E or $\mathrm{N}$ proteins from medium or cell samples were quantified by scanning the band densities from immunoblots. Ratios of HA-E $\triangle 76 \mathrm{~V}$ in media to those in cells were determined for each samples and normalized to that of HA-E in parallel experiment. The level of $\mathrm{N}$-associated VLP $(\mathrm{M}+\mathrm{N})$ production is determined as described in the legend to Figure 1. (C) Removal of the last E carboxyl-terminal residue significantly affected E/N interaction. 293 T cells were co-transfected with HA-tagged wt or $\Delta 76 \mathrm{~V}$ and GST, GST-M or GST-N expression vectors. GST-N and GST-M have GST tags at the amino-terminals of the SARS-CoV N and M coding sequences, respectively. At $48 \mathrm{~h}$ post-transfection, cells and supernatants were collected and prepared for protein analysis. Aliquots of cell lysate samples were subjected to GST pull down analyses (right-hand panel).

be formed by $\mathrm{M}$ plus $\mathrm{N}, \mathrm{M}$ plus $\mathrm{E}, \mathrm{E}$ plus $\mathrm{N}$ or $\mathrm{M}$ plus $\mathrm{N}$ and $\mathrm{E}$. It is likely that there may be a combination of these different VLPs in culture medium when cells are co-transfected with $\mathrm{M}$ plus $\mathrm{E}$ and $\mathrm{N}$. The iodixanol density gradient fractionation analyses suggest that released E vesicles and E-N VLPs exhibit slightly higher densities compared to those of M-E-N VLPs (Figure 2C). Since $\mathrm{M}$ is the major viral component, the strong presence of $\mathrm{M}$ molecules may exert a spatial effect that explains, at least in part, the lower density for M-E-N particles. We consistently observed marked E-N VLP yield enhancement following $M$ co-expression. Since $M$ 


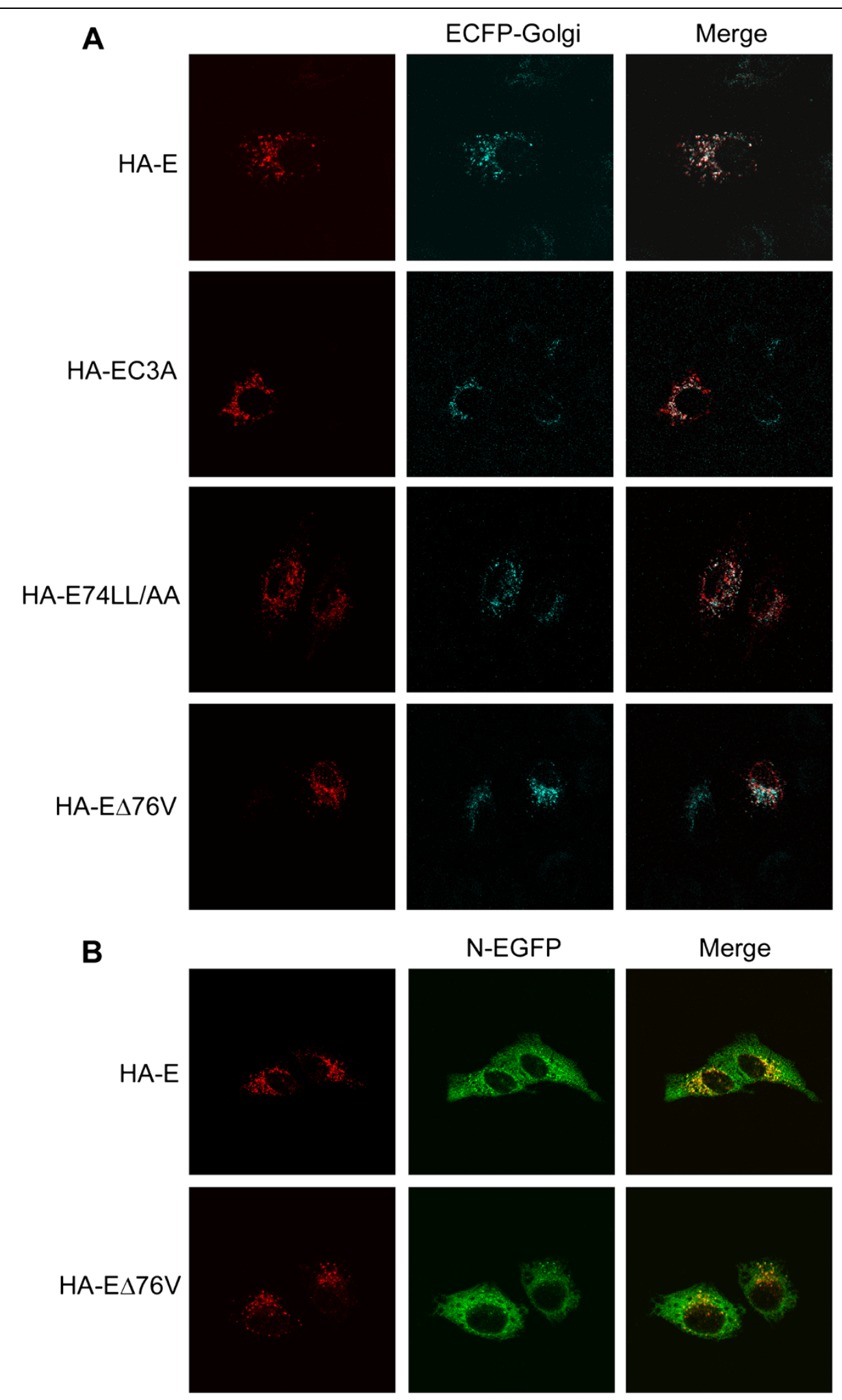



were co-transfected with the indicated HA-tagged wt or mutant E expression vectors plus pGolgi-ECFP, which encodes a Golgi apparatus labeling marker. (B) Co-localization of SARS-CoV E and N proteins. HeLa cells were co-transfected with SARS-CoV N bearing carboxyl-terminal tagged EGFP (N-EGFP) and HA-tagged wt or $\Delta 76 \mathrm{~V}$ expression vectors. At $18 \mathrm{~h}$ post-transfection, cells were fixed and labeled with a primary anti-HA antibody and a secondary rhodamine-conjugated anti-mouse antibody. Images represent the most prevalent phenotypes. Merged red/blue or red/green fluorescent images are shown in right-hand column panels. Mock-transfected cells failed to yield any signal (data not shown).

is the most abundant viral structural protein capable of recruiting both $\mathrm{E}$ and $\mathrm{N}$ into VLPs, we do not consider this a surprising result. In addition, $\mathrm{M}$ exhibits a noticeably higher $\mathrm{N}$ binding capacity than E (Figure 5 C). VLPs formed by $\mathrm{M}, \mathrm{E}$ and $\mathrm{N}$ look more morphologically homogeneous than E-N VLPs, possibly due to additional intermolecular interactions between $\mathrm{M} / \mathrm{M}, \mathrm{M} / \mathrm{E}$ and $\mathrm{M} / \mathrm{N}$.

SARS-CoV E is capable of undergoing oligomerization triggered by both hydrophobic interaction and interchain disulfide bond formation between cysteine residues [16,31]. All three SARS-CoV E cysteine residues have been shown to be post-translationally modified by palmitoylation [16]. Blocking MHV E palmitoylation results in significantly impaired VLP assembly, suggesting that palmitoylated $\mathrm{E}$ proteins are essential for murine coronavirus assembly [14]. In the present study we found that changing all three cysteines into alanines (EC3A) did not exert negative effects on SARS-CoV E protein release or VLP assembly (Figure 4). This suggests that E palmitoylation or interchain disulfide bond formation is not required for SARS-CoV E protein release or VLP assembly. Furthermore, we consistently detected higher levels of EC3A compared to wt E in medium, suggesting that EC3A is released more efficiently than wt E. One possible explanation is that post-translational palmitoylation or interchain disulfide bond formation restricts $\mathrm{E}$ protein secretion.

Despite a higher release level compared to wt E, EC3A did not produce higher VLP yields than wt E following co-expression with $\mathrm{M}$ and $\mathrm{N}$, suggesting no correlation between SARS-CoV E release capacity and its contribution to efficient VLP production. Furthermore, E $\Delta 76 \mathrm{~A}$ was still capable of enhancing VLP production even though it was defective in both release and $\mathrm{N}$ association. Although E $\triangle 76 \mathrm{~A}$ is capable of $\mathrm{M}$ association (as shown by GST assays), it is not efficiently incorporated into VLPs when co-expressed with M. A possible explanation for this discrepancy is that the disruption of cellular compartments allows $\mathrm{E} / \mathrm{M}$ to associate at a higher capacity, whereas less $\mathrm{E} / \mathrm{M}$ association occurs in assembly and budding compartments such as ER/Golgi, resulting in smaller amounts of $E$ being packaged into virions. This scenario is compatible with the proposal that E may promote virus assembly and/or budding by facilitating membrane bending and scissions without required packaging into virions. In support of this hypothesis, one research team has reported that E deletion does not significantly affect SARS-CoV replication, but that E-deleted mutants exhibit 100- to 1,000-fold reductions in virus yields associated with decreased efficiency during morphogenesis [32]. Although E/N interactions may not be directly involved in virus assembly and budding, it remains to be seen whether the SARS$\mathrm{CoV} \mathrm{E}$ contribution to virus production requires efficient $\mathrm{E} / \mathrm{M}$ interaction.

\section{Conclusions}

Palmitoylation or interchain disulfide bond formation appears to be dispensable for the SARS-CoV E enhancement of VLP yields. The contribution of SARS-CoV E to efficient VLP production is also independent of its release capacity, association with $\mathrm{N}$, or VLP incorporation. Additional experiments are required to clarify the biological relevance of SARS-CoV E/N interaction, and to determine whether $\mathrm{E} / \mathrm{N}$ interaction also exists in other coronaviruses.

\section{Competing interests}

The authors declare that they have no competing interests.

\section{Authors' contributions}

CTW designed the experiments and wrote the paper. YTT, SMW and $\mathrm{KJH}$ carried out the experiments and analyzed the data. All authors read and approved the final manuscript.

\section{Acknowledgements}

The authors wish to thank Ming-Wei Guo for reagents and technical assistance. This work was supported by Grants V98C1-021 and V99C1-013 from Taipei Veterans General Hospital, by Grant NSC 97-2320-B-010-002-MY3 and 100-2320-B-010-015-MY3 from the National Science Council, Taiwan, and by a grant from the Ministry of Education, Aim for the Top University Plan.

Received: 15 January 2014 Accepted: 21 April 2014

Published: 27 April 2014

\section{References}

1. Lai MM: Molecular biology of coronavirus 1986. Adv Exp Med Biol 1987, 218:7-13.

2. Masters PS: The molecular biology of coronaviruses. Adv Virus Res 2006, 66:193-292.

3. Klumperman J, Locker JK, Meijer A, Horzinek MC, Geuze HJ, Rottier PJ: Coronavirus $\mathrm{M}$ proteins accumulate in the Golgi complex beyond the site of virion budding. J Virol 1994, 68(10):6523-6534.

4. Tooze J, Tooze S, Warren G: Replication of coronavirus MHV-A59 in sac- cells: determination of the first site of budding of progeny virions. Eur J Cell Biol 1984, 33:281-293.

5. Corse E, Machamer CE: Infectious bronchitis virus E protein is targeted to the Golgi complex and directs release of virus-like particles. J Virol 2000, 74(9):4319-4326. 
6. de Haan CA, Rottier PJ: Molecular interactions in the assembly of coronaviruses. Adv Virus Res 2005, 64:165-230.

7. Hurst KR, Kuo L, Koetzner CA, Ye R, Hsue B, Masters PS: A major determinant for membrane protein interaction localizes to the carboxy-terminal domain of the mouse coronavirus nucleocapsid protein. J Virol 2005, 79(21):13285-13297.

8. Kuo L, Masters PS: Genetic evidence for a structural interaction between the carboxy termini of the membrane and nucleocapsid proteins of mouse hepatitis virus. J Virol 2002, 76(10):4987-4999.

9. Narayanan K, Maeda A, Maeda J, Makino S: Characterization of the coronavirus $M$ protein and nucleocapsid interaction in infected cells. J Virol 2000, 74(17):8127-8134.

10. Risco C, Anton IM, Enjuanes L, Carrascosa JL: The transmissible gastroenteritis coronavirus contains a spherical core shell consisting of M and N proteins. J Virol 1996, 70(7):4773-4777.

11. Sturman LS, Holmes KV, Behnke J: Isolation of coronavirus envelope glycoproteins and interaction with the viral nucleocapsid. J Virol 1980, 33(1):449-462.

12. Wilson L, Gage P, Ewart G: Hexamethylene amiloride blocks E protein ion channels and inhibits coronavirus replication. Virology 2006, 353(2):294-306.

13. Wilson L, McKinlay C, Gage P, Ewart G: SARS coronavirus E protein forms cation-selective ion channels. Virology 2004, 330(1):322-331.

14. Boscarino JA, Logan HL, Lacny JJ, Gallagher TM: Envelope protein palmitoylations are crucial for murine coronavirus assembly. J Virol 2008 82(6):2989-2999.

15. Corse E, Machamer CE: The cytoplasmic tail of infectious bronchitis virus E protein directs Golgi targeting. J Virol 2002, 76(3):1273-1284.

16. Liao Y, Yuan Q, Torres J, Tam JP, Liu DX: Biochemical and functional characterization of the membrane association and membrane permeabilizing activity of the severe acute respiratory syndrome coronavirus envelope protein. Virology 2006, 349(2):264-275.

17. Lopez LA, Riffle AJ, Pike SL, Gardner D, Hogue BG: Importance of conserved cysteine residues in the coronavirus envelope protein. J Virol 2008, 82:3000-3010.

18. Vennema H, Godeke GJ, Rossen JW, Voorhout WF, Horzinek MC, Opstelten DJ, Rottier PJ: Nucleocapsid-independent assembly of coronavirus-like particles by co-expression of viral envelope protein genes. Embo J 1996 15(8):2020-2028

19. Baudoux P, Carrat C, Besnardeau L, Charley B, Laude H: Coronavirus pseudoparticles formed with recombinant $M$ and $E$ proteins induce alpha interferon synthesis by leukocytes. J Virol 1998, 72(11):8636-8643.

20. Hsieh PK, Chang SC, Huang CC, Lee TT, Hsiao CW, Kou YH, Chen IY, Chang CK, Huang TH, Chang MF: Assembly of severe acute respiratory syndrome coronavirus RNA packaging signal into virus-like particles is nucleocapsid dependent. J Virol 2005, 79(22):13848-13855.

21. Huang $Y$, Yang ZY, Kong WP, Nabel GJ: Generation of synthetic severe acute respiratory syndrome coronavirus pseudoparticles: implications for assembly and vaccine production. J Virol 2004, 78(22):12557-12565.

22. Tseng Y-T, Wang S-M, Huang K-J, Lee AIR, Chiang C-C, Wang C-T: Self-assembly of severe acute respiratory syndrome coronavirus membrane protein. J Biol Chem 2010, 285(17):12862-12872.

23. Siu YL, Teoh KT, Lo J, Chan CM, Kien F, Escriou N, Tsao SW, Nicholls JM, Altmeyer R, Peiris JSM, Bruzzone R, Nal B: The M, E, and N structural proteins of the severe acute respiratory syndrome coronavirus are required for efficient assembly, trafficking, and release of virus-like particles. J Virol 2008, 82(22):11318-11330.

24. Maeda J, Maeda A, Makino S: Release of coronavirus E protein in membrane vesicles from virus-infected cells and $E$ protein-expressing cells. Virology 1999, 263(2):265-272.

25. Sambrook J, Russell DW: Molecular cloning: a laboratory manual, ed 3rd Cold Spring Harbor. N.Y.: Cold Spring Harbor Laboratory Press; 2001.

26. Cortes P, Weis-Garcia F, Misulovin Z, Nussenzweig A, Lai J-S, Li G, Nussenzweig $M$, Baltimore D: In vitro V(D)J recombination: signal joint formation. Proc Natl Acad Sci USA 1996, 93(24):14008-14013.

27. Wang S-M, Wang C-T: APOBEC3G cytidine deaminase association with coronavirus nucleocapsid protein. Virology 2009, 388(1):112-120.

28. Shih Y-P, Chen C-Y, Liu S-J, Chen K-H, Lee Y-M, Chao Y-C, Chen Y-MA: Identifying epitopes responsible for neutralizing antibody and DC-SIGN binding on the spike glycoprotein of the severe acute respiratory syndrome coronavirus. J Virol 2006, 80(21):10315-10324
29. Wang S-M, Chang Y-F, Chen Y-M, Wang C-T: Severe acute respiratory syndrome coronavirus nucleocapsid protein confers ability to efficiently produce virus-like particles when substituted for the human immunodeficiency virus nucleocapsid domain. J Biomed Sci 2008, 15(6):719-729.

30. Huang Q, Yu L, Petros AM, Gunasekera A, Liu Z, Xu N, Hajduk P, Mack J, Fesik SW, Olejniczak ET: Structure of the N-terminal RNA-binding domain of the SARS CoV nucleocapsid protein. Biochemistry 2004, 43(20):6059-6063.

31. Liao Y, Lescar J, Tam JP, Liu DX: Expression of SARS-coronavirus envelope protein in Escherichia coli cells alters membrane permeability. Biochem Biophys Res Commun 2004, 325(1):374-380.

32. DeDiego ML, Álvarez E, Almazán F, Rejas MT, Lamirande E, Roberts A, Shieh W-J, Zaki SR, Subbarao K, Enjuanes L: A severe acute respiratory syndrome coronavirus that lacks the $\mathrm{E}$ gene is attenuated in vitro and in vivo. J Virol 2007, 81(4):1701-1713.

doi:10.1186/1423-0127-21-34

Cite this article as: Tseng et al:: SARS-CoV envelope protein palmitoylation or nucleocapid association is not required for promoting virus-like particle production. Journal of Biomedical Science 2014 21:34.

\section{Submit your next manuscript to BioMed Central and take full advantage of:}

- Convenient online submission

- Thorough peer review

- No space constraints or color figure charges

- Immediate publication on acceptance

- Inclusion in PubMed, CAS, Scopus and Google Scholar

- Research which is freely available for redistribution

Submit your manuscript at www.biomedcentral.com/submit
C) Biomed Central 